\title{
Imaging Below Irregular Terrain Using RF Tomography
}

\author{
Lorenzo Lo Monte, Member IEEE, Francesco Soldovieri, Member IEEE, \\ Danilo Erricolo, Senior Member IEEE Michael C. Wicks, Fellow IEEE
}

\begin{abstract}
RF tomography is extended for imaging underground structures and tunnels assuming rough terrain. The theory of RF tomography described in [1] remains applicable, provided that a numerical Green's function is computed. An FFTbased and intrinsically parallel method for obtaining numerical Green's functions is described. This method is corroborated with explicit formulas and implemented for RF tomography. Simulated data computed using an FDTD code is used to demonstrate performance.
\end{abstract}

Index Terms - Inverse Problems, RF Tomography, Ground Penetrating Radar, Green's Functions, Tunnel Detection.

\section{INTRODUCTION}

$\mathrm{R}$ ADIO tomography has been proposed for imaging underground dielectric/conducting anomalies [1]. A system using RF tomography employs a set of low-cost, reconfigurable, narrowband electromagnetic transmitters and receivers placed on top, above, or shallowly buried in the ground at arbitrary positions. This system conceptually operates in three stages. The first stage is dedicated to the configuration of the system: during this stage, sensors accurately identify their position, orientation and time reference. During the second stage, a predetermined subset of transmitters radiates a known polarimetric waveform. The probing wave impinges upon a target (represented in terms of dielectric / conducting anomaly), thus producing scattered fields. Spatially distributed receivers collect samples of the total electric field, excise noise, clutter and the direct path, and store the information concerning the scattered field. Then, a different set of transmitters is activated, or waveforms

This work has been sponsored by the U.S. Air Force Research Laboratory, under contract \# FA-8601-09-F-0024, and by the U.S. Air Force Office of Scientific Research under grant \# FA-9550-05-1-0443.

L. Lo Monte is with General Dynamics Information Technology, Wright-Patterson Air Force Base, Dayton, Ohio, 43441, USA, (e-mail: Lorenzo.lomonte.ctr@wpafb.af.mil).

F. Soldovieri is with the "Istituto per il Rilevamento Elettromagnetico dell'Ambiente" (IREA, Institute for Electromagnetic Sensing of the Environment), Consiglio Nazionale delle Ricerche (CNR, Italian national research council), Naples, Italy. (e-mail: soldovieri.f@irea.cnr.it).

D. Erricolo is with the Department of Electrical and Computer Engineering, University of Illinois at Chicago, Chicago, Illinois, 60607 USA (e-mail: Erricolo@ece.uic.edu)

M. C. Wicks is with the University of Dayton Research Institute, Dayton, Ohio, 45443, USA, (e-mail: michael.wicks@udri.udayton.edu). with different polarization are used. During the third stage, recorded data is relayed to a central station for processing and imaging, (see [1] for a pictorial representation). The system operates using ultra-narrowband, adaptive waveforms, thus ensuring low noise, low dispersion and affordable cost of instrumentation.

To date, RF tomography has been implemented only under conditions when the dyadic Green's function for the sensing problem is provided in analytic form, thus limiting its application to homogeneous space, two half-spaces, and, possibly, layered media with planar interfaces.

The novelty of this work is the extension of RF tomography to include irregular surfaces, by computing the related numerical Green's function using computationally efficient algorithms. This is accomplished via a thorough revision of the forward model, while the inversion procedure is essentially preserved.

This paper is organized as follows: Section II provides a review of the forward model used in RF tomography for underground imaging, and Section III briefly discusses the discretization and inversion process for completeness. Section IV describes a theory to obtain numerical Green's functions using the method of moments. In Section $\mathrm{V}$, the implementation of numerical Green's function for RF tomography is detailed. Finally, Section VI provides FDTD simulations to test the performances of the proposed method.

\section{FORWARD MODEL}

\section{A. Notations and Conventions}

In this paper, the $\exp (-i \omega t)$ time dependence of the fields is assumed. Bolded lower case letters represent vectors, while bolded capital case letters represent matrices. Underscored capital letters represent dyadics. Circumflexed bold letters represent unit vectors.

\section{B. Mathematical Description of the Terrain}

This extended version of RF Tomography applies to the three-dimensional geometry shown in Fig. 1. The interface between air and ground is not planar but it is assumed known. It is beneficial to express the electrical properties of the irregular terrain as the superposition of two contributions. The first contribution is associated with an ideal planar half-space 
with an interface at $z=0$, whose equivalent relative dielectric permittivity is described by a scalar function $H: \square^{3} \rightarrow \square$, the Half-Space equivalent dielectric permittivity function, defined as:

$$
H(\tilde{\mathbf{r}})= \begin{cases}\varepsilon_{D}+i \frac{\sigma_{D}}{\omega \varepsilon_{0}} & \tilde{z}<0 \\ 1 & \tilde{z}>0\end{cases}
$$

where $\omega=2 \pi f$ is the angular frequency, $\varepsilon_{D}$ is the relative dielectric permittivity of the ground, $\sigma_{D}$ is the conductivity of the ground, and $\tilde{\mathbf{r}}=\tilde{x} \hat{\mathbf{x}}+\tilde{y} \hat{\mathbf{y}}+\tilde{z} \hat{\mathbf{z}}$ is a position vector used to describe the terrain.

The second contribution is a volumetric function $Q: \square^{3} \rightarrow \square$ with finite support, denoted as the background contrast function, which represents the deviation in dielectric permittivity and conductivity at point $\tilde{\mathbf{r}}$ between the actual values and the values expected from the ideal half-space geometry $H(\mathbf{r})$. The background contrast function is mathematically described as

$$
Q(\tilde{\mathbf{r}})=\left\{\begin{array}{l}
\varepsilon_{r}(\tilde{\mathbf{r}})-\varepsilon_{D}+i \frac{\sigma(\tilde{\mathbf{r}})-\sigma_{D}}{\omega \varepsilon_{0}} \quad \tilde{z}<0 \\
\varepsilon_{r}(\tilde{\mathbf{r}})-1+i \frac{\sigma(\tilde{\mathbf{r}})}{\omega \varepsilon_{0}} \quad \tilde{z}>0
\end{array},\right.
$$

where $\varepsilon_{r}(\tilde{\mathbf{r}}), \quad \sigma(\tilde{\mathbf{r}})$ are the actual relative dielectric permittivity and the actual conductivity of the terrain within the support of $Q$. The support of $Q$ is assumed bounded, implying that for large $x$ and $y$, the surface becomes flat. Note that $H(\tilde{\mathbf{r}})+Q(\tilde{\mathbf{r}})$ returns the actual electrical properties of the irregular terrain.

Note that both $\varepsilon_{D}, \sigma_{D}$ represent estimated values of the belowground electrical properties. For simplicity, both $\varepsilon_{D}$ and $\sigma_{D}$ are assumed frequency independent: this holds true for common soils when the bandwidth available is restricted between the ranges of $0.5 \mathrm{MHz}-10 \mathrm{MHz}$, which is suitable for tunnel detection. However, the following theory can be easily extended to the case in which both $\varepsilon_{D}$ and $\sigma_{D}$ are frequency dependent.

\section{Investigation Domain}

The targets (i.e., tunnels or voids) are assumed to reside within the investigation domain $D$, fully belonging to the lower medium. To ease the mathematical derivation, the region $D$ shall not include any point in which the background contrast function $Q(\tilde{\mathbf{r}})$ differs from zero. A point in region $D$ shall be represented with a separate position vector $\overline{\mathbf{r}}=\bar{x} \hat{\mathbf{x}}+\bar{y} \hat{\mathbf{y}}+\bar{z} \hat{\mathbf{z}}$.

For a target-free scenario, the background electrical properties in $D$ are fully described by the quantities $\varepsilon_{D}, \sigma_{D}$; However, the presence of any subsurface structure creates a deviation in dielectric permittivity and conductivity w.r.t. the background. The actual relative dielectric permittivity distribution $\varepsilon_{r O}(\overline{\mathbf{r}})$ and conductivity distribution $\sigma_{O}(\overline{\mathbf{r}})$ inside the investigation domain $D$ are the unknowns of the inverse problem. To reduce the number of unknowns, the inverse problem is recast in terms of an object contrast function $V: \square^{3} \rightarrow \square$, which is more physically related to the presence/absence of a target, defined as

$$
V(\overline{\mathbf{r}})=\varepsilon_{r O}(\overline{\mathbf{r}})-\varepsilon_{D}+i \frac{\sigma_{O}(\overline{\mathbf{r}})-\sigma_{D}}{\omega \varepsilon_{0}}
$$

Physically, when $V(\overline{\mathbf{r}}) \neq 0$ a dielectric or conducting anomaly is present at location $\overline{\mathbf{r}}$.

\section{Scattering Integral Equation}

Sources and receivers are modeled as electrically small (Hertzian) dipoles of length $l$. It is assumed that at the generic $n$-th field measurement:

- The transmitting dipole is located at position $\mathbf{r}_{n}^{a}$, fed with current of magnitude $I_{n}$ and is directed along $\hat{\mathbf{a}}_{n}$.

- The scattered field is collected at the receiving dipole located at position $\mathbf{r}_{n}^{b}$, and directed along $\hat{\mathbf{b}}_{n}$.

- The angular frequency of operation is $\omega_{n}$.

A distinct measurement $n$ of the complex-valued electric field is obtained by varying any value among $\mathbf{r}_{n}^{b}, \hat{\mathbf{b}}_{n}, \mathbf{r}_{n}^{a}, \hat{\mathbf{a}}_{n}, \omega_{n}$. Following the derivations reported in [1], the contrast function $V(\overline{\mathbf{r}})$ can be linearly related to the $n$-th measurement via the relation:

$$
\begin{aligned}
& E_{n}\left(\mathbf{r}_{n}^{b}, \hat{\mathbf{b}}_{n}, \mathbf{r}_{n}^{a}, \hat{\mathbf{a}}_{n}, \omega_{n}\right)=i \omega_{n} \mu_{0} I I\left\{\hat{\mathbf{b}}_{n}^{T} \cdot \mathbf{G}\left(\mathbf{r}_{n}^{b}, \mathbf{r}_{n}^{a}\right) \cdot \hat{\mathbf{a}}_{n}\right. \\
& \left.k_{0 n}^{2} \iiint_{D} \hat{\mathbf{b}}_{n}^{T} \cdot \underline{\mathbf{G}}\left(\mathbf{r}_{n}^{b}, \overline{\mathbf{r}}\right) \cdot \underline{\mathbf{G}}\left(\overline{\mathbf{r}}, \mathbf{r}_{n}^{a}\right) \cdot \hat{\mathbf{a}}_{n} V(\overline{\mathbf{r}}) d \overline{\mathbf{r}}\right\} \\
& +\hat{\mathbf{b}}_{n}^{T} \cdot \mathbf{z}\left(\mathbf{r}_{n}^{b}, \hat{\mathbf{b}}_{n}, \mathbf{r}_{n}^{a}, \hat{\mathbf{a}}_{n}\right)
\end{aligned}
$$

The previous formula summarizes the Forward Model of RF tomography in its simplest form. In (4), $T$ denotes the operation of transposition, $k_{0 n}^{2}=\omega_{n}^{2} \mu_{0} \varepsilon_{0}$, and $\mathbf{z}$ represents a 
vector accounting for everything that is unknown in this formulation, such as the contribution due to high-order Born series, clutter, thermal and external noise. In this paper, only the first-order Born approximation is used, thus limiting the validity of the reconstruction for low to moderate-contrast targets. However, the use of Born approximation was found to be acceptable for the task of imaging belowground targets, i.e., when only a qualitative description of the dielectric distribution in space is sought $[2,3]$. Nevertheless, the model can be extended to higher-order Born series, as described in [4]: this possibility is currently under investigation. In (4) the Green's dyadic $\underline{\mathbf{G}}$ is the dyadic function that solves the partial differential equation

$$
\nabla \times \nabla \times \underline{\mathbf{G}}\left(\mathbf{r}, \mathbf{r}^{\prime}\right)-k^{2}(\mathbf{r}) \underline{\mathbf{G}}\left(\mathbf{r}, \mathbf{r}^{\prime}\right)=\underline{\mathbf{I}} \delta\left(\mathbf{r}-\mathbf{r}^{\prime}\right),
$$

in which $\mathbf{r}$ is the observation point, $\mathbf{r}$ ' is the source point, and the wavenumber $k(\mathbf{r})$ can be evaluated using

$$
k(\mathbf{r})=\omega \sqrt{\mu_{0}\left(\varepsilon_{0} \varepsilon_{r}(\mathbf{r})+i \sigma(\mathbf{r}) /\left(\omega \varepsilon_{0}\right)\right)} .
$$

Analytical expressions of $\underline{\mathbf{G}}\left(\mathbf{r}, \mathbf{r}^{\prime}\right)$ are found only for simple distributions of $k(\mathbf{r})$, such as homogeneous space or layered media with planar interfaces [5-11]. To employ RF tomography for the imaging of any irregular surface, $\underline{\mathbf{G}}\left(\mathbf{r}, \mathbf{r}^{\prime}\right)$ needs to be computed numerically, possibly in a fast and efficient manner. Starting from section IV, algorithms and formulas that address this problem will be discussed and implemented in RF tomography.

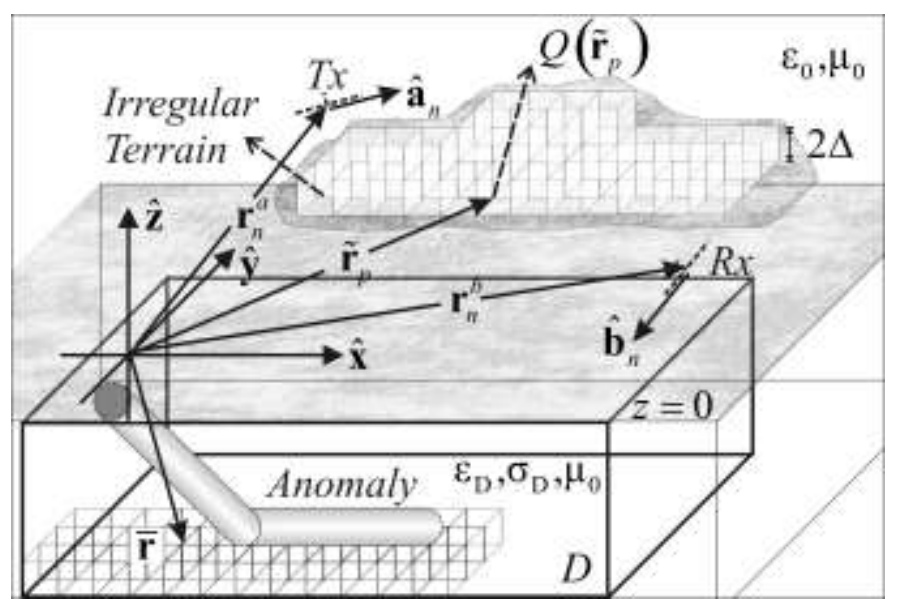

Figure 1: Sample geometry where a portion of irregular surface is shown above the plane $\mathrm{z}=0$. An anomaly, represented by two cylindrical structures, is located underground in region D. One transmitter and one receiver are also indicated together with the directions of their axes.

\section{DISCRETIZATION AND INVERSION}

The continuous linear integral equation in (4) can be discretized using the method of moments. Therefore, a discrete set of $n=1, \ldots, N_{E}$ measurements is collected to form a measurement vector $\mathbf{e}$. The effect of $\mathbf{z}$ is approximately included in $\mathbf{e}$ as a model error (accounting for the higher-order terms in Born series) and noise. The investigation domain $D$ is then discretized in $N_{V}$ voxels, each one located at $\overline{\mathbf{r}}_{d}$, so that the contrast function $V(\overline{\mathbf{r}})$ is recast into a column vector $\mathbf{v}$. The second term in (4) (i.e., the linear integral operation on $V$ ) can be discretized in a matrix $\mathbf{L}$ ( a Dirac $\delta$-function centered at $\overline{\mathbf{r}}_{d}$ is used as the test function) of dimension $N_{E} \times N_{V}$, while the direct path contribution (the term at the right hand of eq.(4)) can be represented by a vector p. Therefore, the forward model can be approximated in discrete form by $\mathbf{e} \cong \mathbf{L} \cdot \mathbf{v}+\mathbf{p}$. In principle, the estimation of $\mathbf{V}$ can be performed as:

$$
\mathbf{v}_{e s t}=\arg \min \|\mathbf{L v}-(\mathbf{e}-\mathbf{p})\|_{2}^{2}+\alpha\|\mathbf{v}\|_{\lambda}
$$

where $\alpha$ is a regularization parameter. This simple estimation problem represents one possible formulation of the inverse model. For example, when $\lambda=2$ eq. (7) reduces to the Tikhonov regularization method [12-13], or when $\lambda=1$, $\mathbf{v}_{e s t}$ is found using sparse regularization methods such as LASSO [14], or FISTA [15-16]. More advanced inversion algorithms might be implemented, such as [17-19], although a reconstruction scheme based on (7) will be used here.

The direct path vector $\mathbf{p}$ is assumed to be perfectly known and completely mitigated. In reality, this is hardly true, and other strategies should be pursued, such as those reported in [20-21].

\section{NUMERICAL GREEN'S FUNCTION}

The application of RF tomography to imaging below irregular terrain according to (4) requires the numerical computation of the Green's function of the problem, which is discussed in this section. Since the topic is applicable beyond RF tomography, a method for finding the value of the numerical Green's function observed at point $\mathbf{r}$, due to a source located at $\mathbf{r}^{\prime}$, at a given angular frequency $\omega$, is addressed at first.

Previous attempts have been made in order to determine the Green's function for rough surfaces, notably by Cui [21] and Akduman [22-23]; however, to the best of the authors' knowledge, no previous paper addresses the computation of numerical 3D Green's functions for irregular surfaces that can be computed using fast and parallel algorithms.

By applying the volume equivalence theorem [24] to $Q(\mathbf{r})$, which is distributed both on top and/or below the half-space surface, the following wave equation can be obtained: 


$$
\begin{gathered}
\nabla \times \nabla \times \mathbf{E}_{S}(\mathbf{r})-k_{H S}^{2}(z) \mathbf{E}_{S}(\mathbf{r})=k_{0}^{2} Q(\mathbf{r}) \mathbf{E}(\mathbf{r}), \\
\mathbf{E}(\mathbf{r})=\mathbf{E}_{S}(\mathbf{r})+\mathbf{E}_{I}(\mathbf{r})
\end{gathered}
$$

In this context, $\mathbf{E}(\mathbf{r})$ represents the total electric field induced by the scattering of the anomaly described by the background contrast function $Q(\mathbf{r}), \mathbf{E}_{S}(\mathbf{r})$ is the scattered field, and $\mathbf{E}_{I}(\mathbf{r})$ is the incident field, $k_{H S}^{2}=k_{0}^{2}, z>0$ and $k_{H S}^{2}=k_{D}^{2}, z<0$, where

$$
\begin{aligned}
& k_{0}=\omega \sqrt{\mu_{0} \varepsilon_{0}}, k_{D}=\omega \sqrt{\mu_{0} \tilde{\varepsilon}_{D}}, \\
& \tilde{\varepsilon}_{D}=\varepsilon_{D}+i \frac{\sigma_{D}}{\omega \varepsilon_{0}}
\end{aligned}
$$

After some manipulations, the solution to equation (8) can be expressed in terms of the scattering contribution $\mathbf{E}_{S}(\mathbf{r})$ :

$$
\begin{aligned}
& \mathbf{E}_{S}(\mathbf{r})=k_{0}^{2} \iiint_{R} Q(\tilde{\mathbf{r}}) \underline{\mathbf{G}}_{H S}(\mathbf{r}, \tilde{\mathbf{r}}) \cdot \mathbf{E}(\tilde{\mathbf{r}}) d \tilde{\mathbf{r}} \\
& =k_{0}^{2} \iiint_{R} Q(\tilde{\mathbf{r}}) \underline{\mathbf{G}}_{H S}(\mathbf{r}, \tilde{\mathbf{r}}) \cdot\left[\mathbf{E}_{S}(\tilde{\mathbf{r}})+\mathbf{E}_{I}(\tilde{\mathbf{r}})\right] d \tilde{\mathbf{r}}
\end{aligned}
$$

where $\underline{\mathbf{G}}_{H S}$ is the half-space dyadic Green's function (analytically known), and $R$ is the support of $Q$.

With some simple steps described in the Appendix, eq. (11) can be generalized in a dyadic form:

$$
\begin{aligned}
\underline{\mathbf{G}}_{S}\left(\mathbf{r}, \mathbf{r}^{\prime}\right)= & k_{0}^{2} \iiint_{R} Q(\tilde{\mathbf{r}}) \underline{\mathbf{G}}_{H S}(\mathbf{r}, \tilde{\mathbf{r}}) . \\
& {\left[\underline{\mathbf{G}}_{S}\left(\tilde{\mathbf{r}}, \mathbf{r}^{\prime}\right)+\underline{\mathbf{G}}_{H S}\left(\tilde{\mathbf{r}}, \mathbf{r}^{\prime}\right)\right] d \tilde{\mathbf{r}} }
\end{aligned}
$$

Eq. (12) can be interpreted as the Green's function representation of the classical scattering integral equation defined in (11). This result can be considered a generalization of the work done by Akduman [22-23] for the 3D case. Clearly, substituting (25), (28) into (9) the Green's function for the irregular surface is obtained:

$$
\underline{\mathbf{G}}\left(\mathbf{r}, \mathbf{r}^{\prime}\right)=\underline{\mathbf{G}}_{H S}\left(\mathbf{r}, \mathbf{r}^{\prime}\right)+\underline{\mathbf{G}}_{S}\left(\mathbf{r}, \mathbf{r}^{\prime}\right) .
$$

Part of eq. (12) can be computed analytically. Therefore, the left hand side of (12) can be separated into two dyadic functions: the part that can be computed analytically

$$
\underline{\mathbf{G}}_{K}\left(\mathbf{r}, \mathbf{r}^{\prime}\right)=k_{0}^{2} \iiint_{R} Q(\tilde{\mathbf{r}}) \underline{\mathbf{G}}_{H S}(\mathbf{r}, \tilde{\mathbf{r}}) \cdot \underline{\mathbf{G}}_{H S}\left(\tilde{\mathbf{r}}, \mathbf{r}^{\prime}\right) d \tilde{\mathbf{r}}
$$

and the part requiring numerical computation:

$$
\underline{\mathbf{G}}_{U}\left(\mathbf{r}, \mathbf{r}^{\prime}\right)=k_{0}^{2} \iiint_{R} Q(\tilde{\mathbf{r}}) \underline{\mathbf{G}}_{H S}(\mathbf{r}, \tilde{\mathbf{r}}) \cdot \underline{\mathbf{G}}_{S}\left(\tilde{\mathbf{r}}, \mathbf{r}^{\prime}\right) d \tilde{\mathbf{r}}
$$

Retrieving $\underline{\mathbf{G}}_{S}\left(\tilde{\mathbf{r}}, \mathbf{r}^{\prime}\right)$ from (15) is difficult, and an approximation is needed. Similarly, the computation of (14) involves at least a 4D Fourier transformation [5], which dramatically impacts the performance.

In both cases, the computation is facilitated by discretizing the region $R$ in $P$ small cubes (voxels), representing an orthogonal basis expansion for $Q$. Each cube $p$ is centered at position $\tilde{\mathbf{r}}_{p}$, whose volume $R_{p}$ is defined as:

$$
R_{p}=\left\{\begin{array}{l}
\tilde{x}_{p}-\Delta \leq \tilde{x} \leq \tilde{x}_{p}+\Delta \\
\tilde{y}_{p}-\Delta \leq \tilde{y} \leq \tilde{y}_{p}+\Delta \\
\tilde{z}_{p}-\Delta \leq \tilde{z} \leq \tilde{z}_{p}+\Delta
\end{array}\right.
$$

where $\Delta$ is the half size of the elementary cube. The dimension of the voxel is chosen so that $\underline{\mathbf{G}}_{H S}\left(\tilde{\mathbf{r}}_{p}, \mathbf{r}^{\prime}\right)$ and $\underline{\mathbf{G}}_{S}\left(\tilde{\mathbf{r}}_{p}, \mathbf{r}^{\prime}\right)$ can be assumed constant within the boundaries of the voxel itself.

Accordingly, (14) and (15) can be discretized as follows:

$$
\begin{aligned}
& \underline{\mathbf{G}}_{U}\left(\mathbf{r}, \mathbf{r}^{\prime}\right) \cong k_{0}^{2} \sum_{p=1}^{P} Q\left(\tilde{\mathbf{r}}_{p}\right) \underline{\mathbf{S}}\left(\mathbf{r}, \tilde{\mathbf{r}}_{p}\right) \cdot \underline{\mathbf{G}}_{S}\left(\tilde{\mathbf{r}}_{p}, \mathbf{r}^{\prime}\right) \\
& \underline{\mathbf{G}}_{K}\left(\mathbf{r}, \mathbf{r}^{\prime}\right) \cong k_{0}^{2} \sum_{q=1}^{P} Q\left(\tilde{\mathbf{r}}_{q}\right) \underline{\mathbf{S}}\left(\mathbf{r}, \tilde{\mathbf{r}}_{q}\right) \cdot \underline{\mathbf{G}}_{H S}\left(\tilde{\mathbf{r}}_{q}, \mathbf{r}^{\prime}\right)
\end{aligned}
$$

where the index $q$ has the same meaning of $p$, but is distinguished to emphasize that the summations are computed separately. In (17)-(18) the dyadic expression $\underline{\mathbf{S}}\left(\mathbf{r}, \mathbf{r}^{\prime}\right)$ represents the integral of the half-space Green's function with respect to the source point $\mathbf{r}^{\prime}$, performed over a cubic region having volume $R_{p}$, centered along the source point and computed at the observation point $\mathbf{r}$. The exact evaluation of this integral is described in Appendix C.

The integral equation (12) can be discretized as:

$$
\begin{aligned}
& \underline{\mathbf{G}}_{S}\left(\mathbf{r}, \mathbf{r}^{\prime}\right)- \\
& k_{0}^{2} \sum_{p=1}^{P} Q\left(\tilde{\mathbf{r}}_{p}\right) \underline{\mathbf{S}}\left(\mathbf{r}, \tilde{\mathbf{r}}_{p}\right) \cdot \underline{\mathbf{G}}_{S}\left(\tilde{\mathbf{r}}_{p}, \mathbf{r}^{\prime}\right)=\underline{\mathbf{G}}_{K}\left(\mathbf{r}, \mathbf{r}^{\prime}\right)
\end{aligned}
$$

In this equation, $\underline{G}_{S}\left(\mathbf{r}, \mathbf{r}^{\prime}\right)$ would be determined only if the value of $\underline{\mathbf{G}}_{S}\left(\tilde{\mathbf{r}}_{p}, \mathbf{r}^{\prime}\right)$ is known for any value of $p$.To find 
$\underline{\mathbf{G}}_{S}\left(\tilde{\mathbf{r}}_{p}, \mathbf{r}^{\prime}\right) \forall p$, eq. (19) is tested exactly at the locations $\tilde{\mathbf{r}}_{p}$, leading to the following set of $P$ equations:

$$
\begin{aligned}
& \underline{\mathbf{G}}_{S}\left(\tilde{\mathbf{r}}_{p}, \mathbf{r}^{\prime}\right)-k_{0}^{2} \sum_{q=1}^{P} Q\left(\tilde{\mathbf{r}}_{q}\right) \underline{\mathbf{S}}\left(\tilde{\mathbf{r}}_{p}, \tilde{\mathbf{r}}_{q}\right) \cdot \underline{\mathbf{G}}_{S}\left(\tilde{\mathbf{r}}_{q}, \mathbf{r}^{\prime}\right) \\
& =\underline{\mathbf{G}}_{K}\left(\tilde{\mathbf{r}}_{p}, \mathbf{r}^{\prime}\right) \quad p=1 \ldots P
\end{aligned}
$$

Due to the dyadic nature of $(20), 9 P$ equations with $9 P$ unknowns can be defined, leading to the creation of the matrix equation:

$$
\mathbf{A x}=\mathbf{g}
$$

The actual entries of $\mathbf{A}, \mathbf{x}$ and $\mathbf{g}$ are described in Appendix B. By solving $\mathbf{X}$ for all dyadic components, one can finally compute the Green's dyadic due to the background contrast function as:

$$
\begin{aligned}
\underline{\mathbf{G}}_{S}\left(\mathbf{r}, \mathbf{r}^{\prime}\right)= & \underline{\mathbf{G}}_{K}\left(\mathbf{r}, \mathbf{r}^{\prime}\right)+ \\
& k_{0}^{2} \sum_{p=1}^{P} Q\left(\tilde{\mathbf{r}}_{p}\right) \underline{\mathbf{S}}\left(\mathbf{r}, \tilde{\mathbf{r}}_{p}\right) \cdot \underline{\mathbf{G}}_{S}\left(\tilde{\mathbf{r}}_{p}, \mathbf{r}^{\prime}\right)
\end{aligned}
$$

The final numerical Green's dyadic can be computed by substituting (22) in (13).

\section{APPLICATION TO RF TOMOGRAPHY}

Following the forward model described in (4), three kinds of numerical Green's functions need to be computed for each measurement $n$ :

- $\mathbf{G}\left(\overline{\mathbf{r}}, \mathbf{r}_{n}^{a}\right)$ : the observation point $\overline{\mathbf{r}}$ spans the $x-y$ plane (constant $\bar{z}$ ) where the subsurface anomaly is expected, while $\mathbf{r}_{n}^{a}$ is kept fixed.

- $\underline{\mathbf{G}}\left(\mathbf{r}_{n}^{b}, \overline{\mathbf{r}}\right)$ : the source point $\overline{\mathbf{r}}$ spans the $x$ - $y$ plane (constant $\bar{z}$ ) where the subsurface anomaly is expected, while $\mathbf{r}_{n}^{b}$ is kept fixed.

- $\underline{\mathbf{G}}\left(\mathbf{r}_{n}^{b}, \mathbf{r}_{n}^{a}\right)$ : the Green's function is simply computed pointto-point.

For the first, second and third kind of Green's function, a matrix equation is constructed substituting $\mathbf{r}^{\prime}=\mathbf{r}_{n}^{a}, \overline{\mathbf{r}}, \mathbf{r}_{n}^{a}$ into (20), respectively. Note that the resulting matrix $\mathbf{A}$ is equal for all Green's functions, so it needs to be computed and inverted only once (at constant frequency), while the vector $\mathbf{g}$

in (21) can be computed by substituting $\mathbf{r}^{\prime}=\mathbf{r}_{n}^{a}, \overline{\mathbf{r}}, \mathbf{r}_{n}^{a}$ into (18), respectively. Although $\mathbf{A}$ can be very large, it is square and full rank: hence, the inversion can be accelerated using fast Gaussian reduction [25] or similar algorithms in which only one row of $\mathbf{A}$ needs to be processed per iteration. After obtaining $\mathbf{x}$ from (21), the corresponding $\underline{\mathbf{G}}_{S}\left(\tilde{\mathbf{r}}_{p}, \mathbf{r}^{\prime}\right)$ are evaluated $\forall p$. Then, the numerical Green's function is easily computed as:

$$
\begin{aligned}
& \underline{\mathbf{G}}\left(\mathbf{r}, \mathbf{r}^{\prime}\right)=\underline{\mathbf{G}}_{H S}\left(\mathbf{r}, \mathbf{r}^{\prime}\right)+ \\
& k_{0}^{2} \sum_{p=1}^{P} Q\left(\tilde{\mathbf{r}}_{p}\right) \underline{\mathbf{S}}\left(\mathbf{r}, \tilde{\mathbf{r}_{p}}\right) \cdot\left[\underline{\mathbf{G}}_{H S}\left(\tilde{\mathbf{r}}_{p}, \mathbf{r}^{\prime}\right)+\underline{\mathbf{G}}_{S}\left(\tilde{\mathbf{r}}_{p}, \mathbf{r}^{\prime}\right)\right]
\end{aligned}
$$

where $\mathbf{r}=\overline{\mathbf{r}}, \mathbf{r}_{n}^{b}, \mathbf{r}_{n}^{b}$ and $\mathbf{r}^{\prime}=\mathbf{r}_{n}^{a}, \overline{\mathbf{r}}, \mathbf{r}_{n}^{a}$, respectively. When this algorithm is translated into software, one quickly realizes that most steps are easily parallelized, and may be computed in a few iterations. In Appendix $\mathrm{C}$, the computation of the Green's function in a single horizontal "snapshot" using FFT routines is discussed and described.

\section{SIMULATIONS}

A large-scale numerical simulation has been performed to prove the validity of this novel method. A half-space geometry is considered and, to simulate irregular terrain, a parallelepiped of size $32 \mathrm{~m} \times 32 \mathrm{~m} \times 8 \mathrm{~m}$ with the same properties of the ground is placed on top of the flat surface at the center of the scene (see Fig.2). Five transmitters and receivers are distributed uniformly along a circle of radius 15 $\mathrm{m}$, at $0.5 \mathrm{~m}$ above the box, to avoid numerical instabilities. The target is an inverse- $\mathrm{L}$ shaped tunnel of equal sides and radius $1 \mathrm{~m}$, located at depth of $10 \mathrm{~m}$, as shown in Fig. 3. The frequency range is $3-6 \mathrm{MHz}$, at constant intervals of $1 \mathrm{MHz}$. The electrical properties of the soil are chosen to be similar to dry rocks [35-36]; accordingly, the background dielectric permittivity is $\varepsilon_{D}=10$ and the background dielectric conductivity is $\sigma_{D}=5 \times 10^{-4} \mathrm{~S} / \mathrm{m}$. To avoid the so-called "inverse problem crime", the corresponding electric field at the receiver side has been computed numerically using the Finite Difference Time Domain (FDTD) code GPRMAX [37]. For the FDTD solver, the discretization step is $d=0.25 \mathrm{~m}$, and the box size containing the whole scene is $150 \mathrm{~m} \times 150 \mathrm{~m} \times 120 \mathrm{~m}$.

For each Tx-Rx-frequency combination, two simulations were performed: one containing the target (i.e., the total field), and one without (i.e., the background). The scattered field in complex form can be obtained by subtracting these two timedomain data sets, followed by an FFT (see [1] for details). For such a large-scale simulation, 250 hours of CPU time were required just to solve the forward problem, i.e., to create the measurement vector $\mathbf{e}$.

To fill $\mathbf{L}$, the algorithm proposed in this paper was applied. The irregular surface (i.e., the flat surface with the box on top) was discretized in cubes having $\Delta=2 \mathrm{~m}$, corresponding to $P=128$, which is moderately coarse but manageable in terms of memory allocation. Better performances is expected 
using smaller $\Delta$, but more sophisticated and memory-saving algorithms required to fill and invert $\mathbf{A}$. The region of interest $D$ was divided in three horizontal slices located at depth $-15 \mathrm{~m}$, $-10 \mathrm{~m}$ and $-5 \mathrm{~m}$, each one discretized using a cubic mesh of half size of $\delta=0.5 \mathrm{~m}$; Both the layered visualization and the $2 \mathrm{D}$ relevant slice at depth of $-10 \mathrm{~m}$ are presented. The direct path was eliminated a priori since its value was accurately estimated using (4). The data is noiseless; however, discrepancies between the numerical FDTD solver and the proposed MoM-based method can be assumed as unknown perturbation of the measured field, thus implicitly testing the robustness of our algorithm with respect to disturbances. Further information can be found in [1], where a discussion of why thermal white Gaussian noise is not a main concern in RF tomography is presented.

The reconstruction was performed using a modified FISTA algorithm [15], which solves the following minimization problem:

$$
\begin{aligned}
& \mathbf{v}_{\text {est }}=\arg \min \|\mathbf{L v}-(\mathbf{e}-\mathbf{p})\|_{2}^{2}+\alpha\|\mathbf{v}\|_{1} \\
& \text { subject to: }\left|(\mathbf{v})_{\chi}\right| \leq\left|\widetilde{\varepsilon}_{D}\right| \quad \forall \chi
\end{aligned}
$$

where $(\mathbf{v})_{\chi}$ indicates the $\chi$-th element of $\mathbf{v}$. This minimization guarantees a sparse solution having the physical meaning that each voxel in $D$ cannot be (in absolute value) greater than the background itself. This constraint is particularly useful for tunnel detection, where the maximum difference in electrical properties between the anomaly and the background cannot exceed $\left|\tilde{\varepsilon}_{D}\right|$ in principle. The regularization parameter $\alpha$ is heuristically determined and it does not have to be the same for different simulations since it depends on the entries of matrix $\mathbf{L}$.

However, other methods could be used, such as the ones described in [1-4,34,38,39].

The reconstructed image of the belowground surface is shown in Figs. 4-5. In Figs. 6-7, a reconstruction was performed for the same irregular geometry in Fig.2 but using the analytical half-space Green's function in the reconstruction scheme. By comparing Figs. 4-5 with Figs. 6-7 one clearly notices the advantage of using a numerical Green's function versus the simplified half-space geometry.

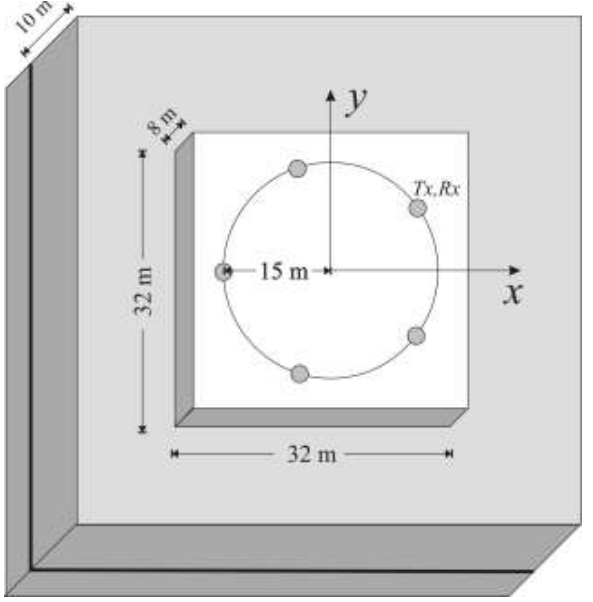

Figure 2: Geometry consisting of a flat surface with a parallelepiped on top of that is considered in the numerical simulation

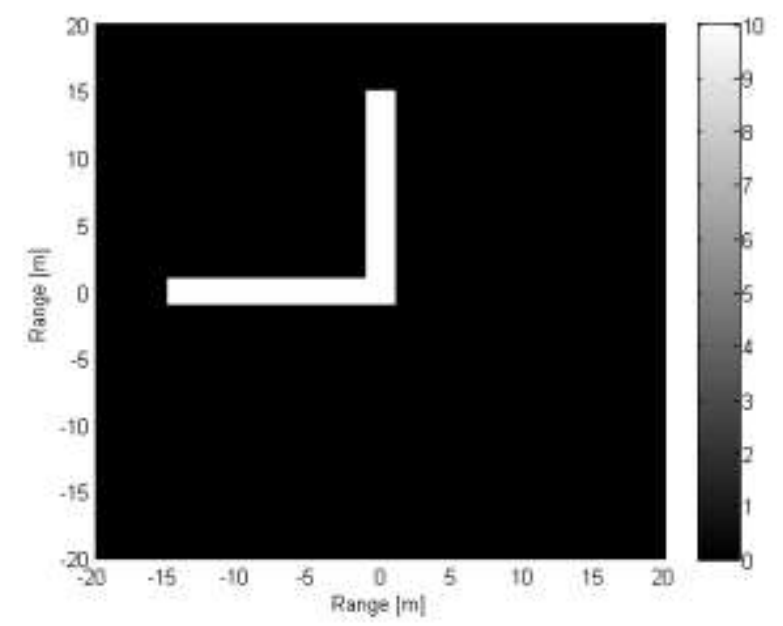

Figure 3: Actual shape of the anomaly $V$ that is used by GPRMAX to compute the measurement vector $\mathbf{e}$. The depth

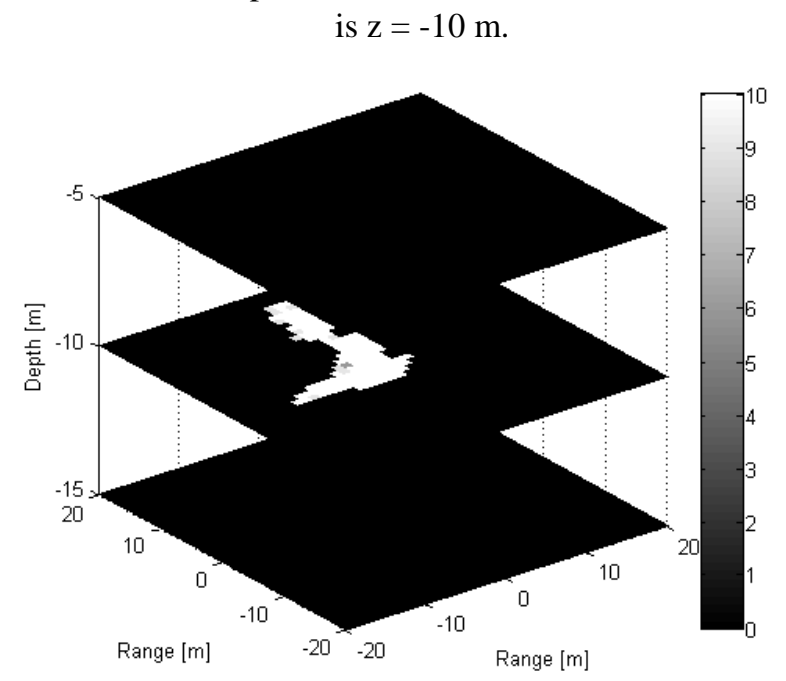

Figure 4. Magnitude $|V(\overline{\mathbf{r}})|$ obtained using the numerical Green's function at three values of depth: $z=-5 \mathrm{~m} \mathrm{z}=-10 \mathrm{~m}$ and $\mathrm{z}=-15 \mathrm{~m}$. 


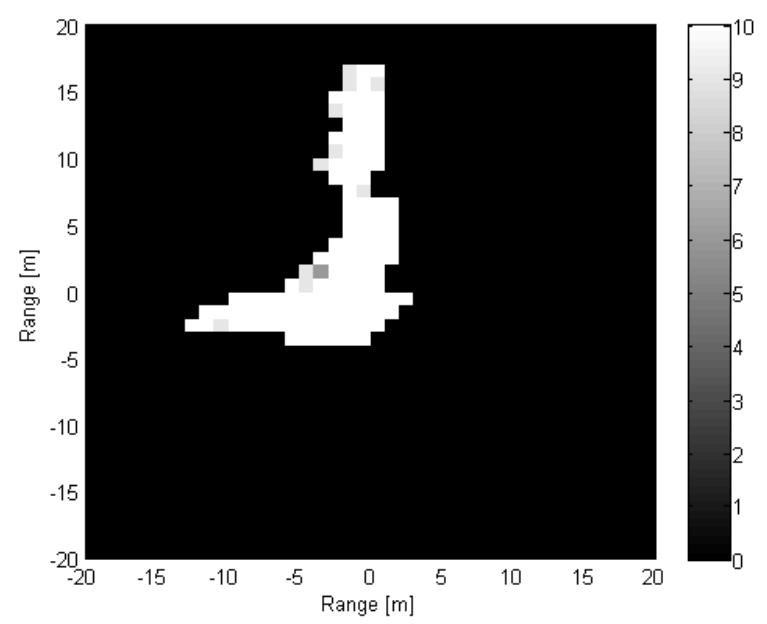

Figure 5: Magnitude $|V(\overline{\mathbf{r}})|$ using numerical Green's for a slice at a depth of $\mathrm{z}=-10 \mathrm{~m}$

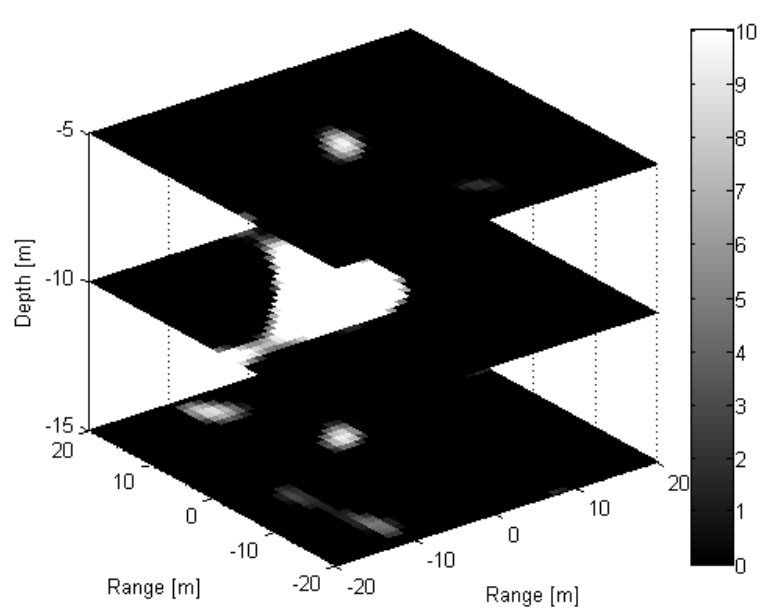

Figure 6: Magnitude $|V(\overline{\mathbf{r}})|$ obtained using the half-space

Green's function in the reconstruction procedure for three values of depth: $\mathrm{z}=-15 \mathrm{~m}, \mathrm{z}=-10 \mathrm{~m}, \mathrm{z}=-15 \mathrm{~m}$. The measured data was generated assuming the actual irregular terrain shape in Fig. 2.

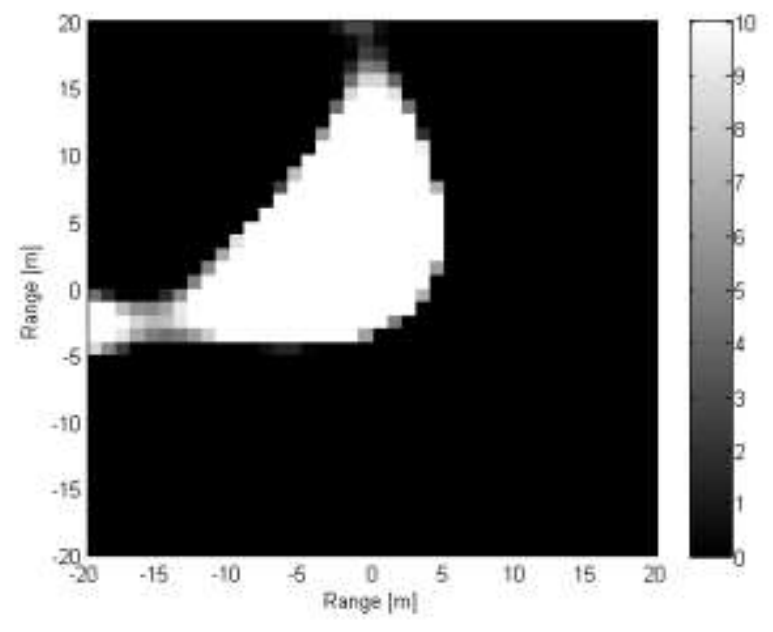

Figure 7: Detail of the magnitude $|V(\overline{\mathbf{r}})|$ obtained using the half-space Green's function in the reconstruction procedure, when the measured field $\mathbf{e}$ was generated considering the irregular terrain

\section{CONCLUSION}

$\mathrm{RF}$ tomography for underground imaging is generalized to address the case in which the terrain shape is irregular but known.

This effort was motivated by the fact that very rarely perfectly flat surfaces are encountered in nature, and the overlysimplified assumption of using half-space geometries yield inconsistent reconstruction results.

Previous work characterized the rigorous scattering from irregular surfaces, but its main focus was the description of the forward model, neglecting the implementation for inverse scattering algorithms. Few authors have attempted to apply this knowledge to an imaging problem, and their derivation can be applied only to a limited set of cases (for example, simplified 2D scenario with plane waves, 3D scenario with PEC surface, or using a statistical description for the roughness of the terrain [40-41]). Differently from previous works, this method is entirely focused on subsurface imaging of dielectric and conducting targets (i.e., no PEC surface assumption), uses 3D vector formulas that are valid also in the near field, includes shading and occlusion effects, and partially account for the radiation pattern of the sensor. Moreover, the algorithm is carefully designed to be computed using standard FFT techniques, and it is very suited for parallelization. There are still some challenges to be overcome, such as the fast growth of the size of $\mathbf{A}$, the time to fill $\mathbf{L}$, the possibility of having $Q(\tilde{\mathbf{r}}) \neq 0$ in $D$, as well as the numerous computations of various $\underline{\mathbf{G}}_{K}$, or the analytical derivation of other integrals of Green's functions, particularly for the magnetic field and for magnetic sources in layered media. Furthermore, the inverted model errors, particularly w.r.t. the error on the interface shape and the background electrical properties, need to be thoroughly investigated. These problems are currently under investigation.

\section{APPENDIX}

\section{A. MoM Extension to Green's Dyadics}

By inspecting (11), each field contribution can be assumed to be generated by an arbitrary source located at $\mathbf{r}^{\prime}$ having dipole moment $I_{0} l \neq 0$ and direction $\hat{\mathbf{a}}$ :

$$
\begin{aligned}
& \mathbf{E}_{S}(\mathbf{r})=i \omega \mu_{0} I_{0} l \underline{G}_{S}\left(\mathbf{r}, \mathbf{r}^{\prime}\right) \cdot \hat{\mathbf{a}} \\
& \mathbf{E}_{S}(\tilde{\mathbf{r}})=i \omega \mu_{0} I_{0} l \underline{G}_{S}\left(\tilde{\mathbf{r}}, \mathbf{r}^{\prime}\right) \cdot \hat{\mathbf{a}} \\
& \mathbf{E}_{I}(\tilde{\mathbf{r}})=i \omega \mu_{0} I_{0} l \underline{\mathbf{G}}_{H S}\left(\tilde{\mathbf{r}}, \mathbf{r}^{\prime}\right) \cdot \hat{\mathbf{a}}
\end{aligned}
$$




$$
\mathbf{E}_{I}(\mathbf{r})=i \omega \mu_{0} I_{0} l \underline{\mathbf{G}}_{H S}\left(\mathbf{r}, \mathbf{r}^{\prime}\right) \cdot \hat{\mathbf{a}}
$$

In these formulas, $\underline{\mathbf{G}}_{S}$ is a dyadic contribution due to the presence of a non-zero background contrast function. Substituting (25)-(27) into (11), and dividing by $i \omega \mu_{0} I_{0} l$, respectively, eq. (11) can be expressed in dyadic form:

$$
\begin{array}{r}
\left\{\underline{\mathbf{G}}_{S}\left(\mathbf{r}, \mathbf{r}^{\prime}\right)-k_{0}^{2} \iiint_{R} Q(\tilde{\mathbf{r}}) \underline{\mathbf{G}}_{H S}(\mathbf{r}, \tilde{\mathbf{r}}) \cdot\right. \\
\left.\left[\underline{\mathbf{G}}_{S}\left(\tilde{\mathbf{r}}, \mathbf{r}^{\prime}\right)+\underline{\mathbf{G}}_{H S}\left(\tilde{\mathbf{r}}, \mathbf{r}^{\prime}\right)\right] d \tilde{\mathbf{r}}\right\} \cdot \hat{\mathbf{a}}=\mathbf{0}
\end{array}
$$

Eq. (29) can be recast as the following problem:

$$
\underline{\mathbf{M}} \cdot \hat{\mathbf{a}}=\mathbf{0} \quad \forall \hat{\mathbf{a}} \in \square^{3}
$$

This condition requires that (30) is satisfied if and only if all eigenvalues of $\underline{\mathbf{M}}$ are zero, or in other terms, $\underline{\mathbf{M}}=\underline{\mathbf{0}}$.

Hence, by enforcing (30) on (29), equation (12) is obtained.

\section{B. Entries in the Matrix Equation}

The entries of the matrix equation:

$$
\mathbf{A x}=\mathbf{g}
$$

are described as follows. By appropriately arranging the terms in (20), matrix $\mathbf{A}$ can be expressed as:

$$
\mathbf{A}=\left[\begin{array}{cccc}
\mathbf{I}_{9 \times 9}-\mathbf{Q}^{11} & -\mathbf{Q}^{12} & \cdots & -\mathbf{Q}^{1 P} \\
-\mathbf{Q}^{21} & \mathbf{I}_{9 \times 9}-\mathbf{Q}^{22} & & \\
\vdots & & \ddots & \\
-\mathbf{Q}^{1 P} & & & \mathbf{I}_{9 \times 9}-\mathbf{Q}^{P P}
\end{array}\right]
$$

where $\mathbf{I}_{N \times N}$ is a $N \times N$ identity matrix, and $\mathbf{Q}$ is

$$
\mathbf{Q}^{p q}=k_{0}^{2} Q\left(\tilde{\mathbf{r}}_{q}\right)\left[\begin{array}{lll}
\mathbf{I}_{3 \times 3} S_{x x}^{p q} & \mathbf{I}_{3 \times 3} S_{x y}^{p q} & \mathbf{I}_{3 \times 3} S_{x z}^{p q} \\
\mathbf{I}_{3 \times 3} S_{y x}^{p q} & \mathbf{I}_{3 \times 3} S_{y y}^{p q} & \mathbf{I}_{3 \times 3} S_{y z}^{p q} \\
\mathbf{I}_{3 \times 3} S_{z x}^{p q} & \mathbf{I}_{3 \times 3} S_{z y}^{p q} & \mathbf{I}_{3 \times 3} S_{z z}^{p q}
\end{array}\right],
$$

where $S_{i j}^{p q}$ is the $i j$-th element of the $\underline{\mathbf{S}}\left(\tilde{\mathbf{r}}_{p}, \tilde{\mathbf{r}}_{q}\right)$ dyadic. The explicit expression for $\underline{\mathbf{S}}$ is discussed in Appendix C. The remaining two vectors can be written as

$$
\mathbf{x}=\left[\begin{array}{c}
G_{S}^{x x}\left(\tilde{\mathbf{r}}_{1}, \mathbf{r}^{\prime}\right) \\
G_{S}^{x y}\left(\tilde{\mathbf{r}}_{1}, \mathbf{r}^{\prime}\right) \\
\vdots \\
G_{S}^{z z}\left(\tilde{\mathbf{r}}_{q-1}, \mathbf{r}^{\prime}\right) \\
G_{S}^{x x}\left(\tilde{\mathbf{r}}_{q}, \mathbf{r}^{\prime}\right) \\
\vdots \\
G_{S}^{z z}\left(\tilde{\mathbf{r}}_{P}, \mathbf{r}^{\prime}\right)
\end{array}\right], \quad \mathbf{g}=\left[\begin{array}{c}
G_{K}^{x x}\left(\tilde{\mathbf{r}}_{1}, \mathbf{r}^{\prime}\right) \\
G_{K}^{x y}\left(\tilde{\mathbf{r}}_{1}, \mathbf{r}^{\prime}\right) \\
\vdots \\
G_{K}^{z z}\left(\tilde{\mathbf{r}}_{p-1}, \mathbf{r}^{\prime}\right) \\
G_{K}^{x x}\left(\tilde{\mathbf{r}}_{p}, \mathbf{r}^{\prime}\right) \\
\vdots \\
G_{K}^{z z}\left(\tilde{\mathbf{r}}_{P}, \mathbf{r}^{\prime}\right)
\end{array}\right]
$$

where $G_{S}^{i j}$ and $G_{K}^{i j}$ are the $i j$-th element of the $\underline{\mathbf{G}}_{S}$ and $\underline{\mathbf{G}}_{K}$ dyadics, respectively.

\section{Integral of Half-Space Green's Functions}

The integral of the half-space Green's function $\underline{\mathbf{S}}\left(\mathbf{r}, \mathbf{r}^{\prime}\right)$ over a cube, and the half-space Green's function $\underline{\mathbf{G}}_{H S}\left(\mathbf{r}, \mathbf{r}^{\prime}\right)$, are generally much more difficult to compute compared to the free-space case (throughout this Section, $\mathbf{r}$ is the observation point, while $\mathbf{r}$ 'is the source location). Here, formulas are provided for the integral of the half-space Green's function that are appropriate for fast computation and suit the needs of $\mathrm{RF}$ tomography in which horizontal slices of the underground scene are reconstructed. The integral of the half-space Green's function is intentionally expressed in a 2D spectral-like form, so that 2D-FFT algorithms are applicable. The intermediate steps are omitted, although relevant citations have been properly included. The integral over a cube centered over $\mathbf{r}^{\prime}$ of the half-space dyadic Green's function can be expressed by the following formula:

$$
\underline{\mathbf{S}}\left(\mathbf{r}, \mathbf{r}^{\prime}\right)=\int_{x^{\prime}-\Delta}^{x^{\prime}+\Delta} \int_{y^{\prime}-\Delta}^{y^{\prime}+\Delta} \int_{z^{\prime}-\Delta}^{z^{\prime}+\Delta} \underline{\mathbf{G}}_{H S}\left(\mathbf{r}, \mathbf{r}^{\prime \prime}\right) d \mathbf{r}^{\prime \prime},
$$

where $\mathbf{r}^{\prime \prime}$ is an auxiliary position vector. The analytical evaluation of (34) depends upon the location of $\mathbf{r}$ and $\mathbf{r}^{\prime}$. Accordingly, four different cases can be defined [10]:

$$
\underline{\mathbf{S}}\left(\mathbf{r}, \mathbf{r}^{\prime}\right)=\underline{\mathbf{S}}^{u v}\left(\mathbf{r}, \mathbf{r}^{\prime}\right) \quad u=a, e \quad v=a, e .
$$

Depending upon the kind of integration, the superscript $u v$ indicates that the observation point is included in the $u$ region, and that the integration around the source point is entirely included in the $v$ region: the two available regions are $a=$ air and $e=$ earth.

For the air-air and earth-earth cases, the solution can be expressed as a superposition of a primary field and a reflected field, as follows:

$$
\underline{\mathbf{S}}^{a a, e e}=\underline{\mathbf{S}}_{P}^{a a, e e}+\underline{\mathbf{S}}_{R}^{a a, e e} .
$$


The computation of the primary field contribution is derived from [5,7,10,26-34] and is given by the following formula, both when the integration is performed including or excluding the singularity:

$$
\underline{\mathbf{S}}_{P}^{a a, e e} \cong\left\{\begin{array}{l}
\overbrace{\frac{4 \pi \zeta}{k_{0, D}^{2}\left[\frac{\sin \left(k_{0, D} \zeta\right)}{k_{0, D} \zeta}-\cos \left(k_{0, D} \zeta\right)\right] \underline{\mathbf{G}}_{P}^{a a, e e}}}^{\text {Integration includes singular point at } \mathbf{r}^{\prime}} \\
\overbrace{\left[\frac{2\left(1-i k_{0, D} \zeta\right) e^{+i k_{0, D} \zeta}-3}{3 k_{0, D}^{2}}\right]}^{\text {Integration excludes singular point at } \mathbf{r}^{\prime}} \\
\zeta=2\left(\frac{3}{4 \pi}\right)^{1 / 3} \Delta
\end{array}\right.
$$

where $\underline{\mathbf{G}}_{P}$ is the Green's function for the homogeneous space, which can be derived explicitly by expanding the derivatives of the following formula [7]

$$
\underline{\mathbf{G}}_{P}^{a a, e e}\left(\mathbf{r}, \mathbf{r}^{\prime}\right)=\left(\underline{\mathbf{I}}+\frac{1}{k_{0, D}^{2}} \nabla \nabla\right)\left(\frac{e^{+i k_{0, D} \mid \mathbf{r}^{\prime} \mathbf{r}_{2}}}{4 \pi\left\|\mathbf{r}-\mathbf{r}^{\prime}\right\|_{2}}\right)
$$

Particular care is necessary when the integration is performed close to the singularity, since (37) arises from the approximation that the volume integral along a cubic region is recast in terms of a volume integral along a spherical region having the same volume of the cubic cell. These near-singular regions can be either integrated numerically, or approximated using smaller cell sizes.

The reflection part in $\underline{\mathbf{S}}$ can be expressed in spectral form as

$$
\begin{gathered}
\underline{\mathbf{S}}_{R}^{a a, e e}= \\
\int_{-\infty}^{+\infty} \int_{-\infty}^{+\infty} A^{a a, e e} \underline{\tilde{\mathbf{G}}}^{a a, e e} e^{+i\left[k_{x}\left(x-x^{\prime}\right)+k_{y}\left(y-y^{\prime}\right)\right]} d k_{x} d k_{y}
\end{gathered}
$$

where $\tilde{\mathbf{G}}^{\text {aa,ee }}$ is the spectral representation of the Green's function, and $A$ is a multiplicative factor defined as:

$$
A^{a a, e e}=\left\{\begin{array}{l}
\frac{i}{\pi^{2} k_{0, D}^{2}} \frac{1}{k_{a z, e z}} e^{(+,-) i k_{a z, e z}\left(z+z^{\prime}\right)} \times \\
\frac{\sin \left(\Delta k_{x}\right) \sin \left(\Delta k_{y}\right) \sinh \left(\Delta k_{a z, e z}\right)}{k_{x} k_{y} k_{a z, e z}} \\
i \frac{e^{(+,-) i k_{a z, e z}\left(z+z^{\prime}\right)}}{8 \pi^{2} k_{0, D}^{2} k_{a z, e z}} \text { no integration }
\end{array}\right.
$$

Note that Eq. (41) can be defined in the whole $k$-space, provided that the following value is used as a limit:

$$
\lim _{k_{x, y} \rightarrow 0} \frac{\sin \left(\Delta k_{x, y}\right)}{k_{x, y}}=\lim _{k_{a z, e z} \rightarrow 0} \frac{\sinh \left(\Delta k_{a z, e z}\right)}{k_{a z, e z}}=\Delta .
$$

After some manipulations (see [26-29] for details), the elements of $\underline{\mathbf{G}}$ can be expressed as follows:

$$
\begin{aligned}
& \tilde{G}_{x x}^{a a, e e}=\frac{k_{0, D}^{2} k_{y}^{2} R_{T E}^{a a, e e}-k_{x}^{2} k_{a z, e z}^{2} R_{T M}^{a a, e e}}{k_{x}^{2}+k_{y}^{2}} \\
& \tilde{G}_{x y}^{a a, e e}=\tilde{G}_{y x}^{a a, e e}=-k_{x} k_{y} \frac{k_{0, D}^{2} R_{T E}^{a a, e e}+k_{a z, e z}^{2} R_{T M}^{a a, e e}}{k_{x}^{2}+k_{y}^{2}} \\
& \tilde{G}_{x z}^{a a, e e}=-\tilde{G}_{z x}^{a a, e e}=(-,+) k_{x} k_{a z, e z} R_{T M}^{a a, e e} \\
& \tilde{G}_{y y}^{a a, e e}=\frac{k_{0, D}^{2} k_{x}^{2} R_{T E}^{a a, e e}-k_{y}^{2} k_{a z, e z}^{2} R_{T M}^{a a, e e}}{k_{x}^{2}+k_{y}^{2}} \\
& \tilde{G}_{y z}^{a a, e e}=-\tilde{G}_{z y}^{a a, e e}=(-,+) k_{y} k_{a z, e z} R_{T M}^{a a, e e} \\
& \tilde{G}_{z z}^{a a, e e}=\left(k_{x}^{2}+k_{y}^{2}\right) R_{T M}^{a a, e e}
\end{aligned}
$$

In these expressions, the Fresnel coefficients in spectral domain have been used:

$$
\begin{aligned}
R_{T E}^{a a, e e} & =\frac{k_{a z, e z}-k_{e z, a z}}{k_{a z}+k_{e z}} \\
R_{T M}^{a a, e e} & =(+,-) \frac{\tilde{\varepsilon}_{D} k_{a z}-k_{e z}}{k_{e z}+\tilde{\varepsilon}_{D} k_{a z}} \\
k_{a z, e z} & =\sqrt{k_{0, D}^{2}-k_{x}^{2}-k_{y}^{2}}
\end{aligned}
$$

The air-earth and earth-air components are defined as:

$$
\begin{gathered}
\underline{\mathbf{S}}^{a e, e a}\left(\mathbf{r}, \mathbf{r}^{\prime}\right)= \\
\int_{-\infty}^{+\infty} \int_{-\infty}^{+\infty} A^{a e, e a} \underline{\mathbf{G}}^{a e, e a} e^{+i\left(k_{x}\left(x-x^{\prime}\right)+k_{y}\left(y-y^{\prime}\right)\right)} d k_{x} d k_{y}
\end{gathered}
$$

where

$$
\begin{gathered}
\tilde{\mathbf{G}}^{a e, e a}= \\
{\left[\begin{array}{ccc}
k_{a z} k_{e z}+k_{y}^{2} & -k_{x} k_{y} & (-,+) k_{x} k_{a z, e z} \\
-k_{x} k_{y} & k_{a z} k_{e z}+k_{x}^{2} & (-,+) k_{y} k_{a z, e z} \\
(-,+) k_{x} k_{e z, a z} & (-,+) k_{y} k_{e z, a z} & k_{x}^{2}+k_{y}^{2}
\end{array}\right]}
\end{gathered}
$$




$$
\begin{aligned}
& \text { and } \\
& A^{a e, e a}=\left\{\begin{array}{l}
\frac{+2 i}{\pi^{2} k_{0}^{2}} \frac{e^{+i k_{a z}\left(z, z^{\prime}\right)-i k_{e z}\left(z^{\prime}, z\right)}}{\left(k_{e z}+\varepsilon_{D} k_{a z}\right)} \times \\
\frac{\sin \left(\Delta k_{x}\right) \sin \left(\Delta k_{y}\right) \sinh \left(\Delta k_{a z, e z}\right)}{k_{x} k_{y} k_{e z, a z}} \\
i \frac{e^{+i k_{a z}\left(z, z^{\prime}\right)-i k_{e z}\left(z^{\prime}, z\right)}}{4 \pi^{2} k_{0}^{2}\left(k_{e z}+\tilde{\varepsilon}_{D} k_{a z}\right)} \text { no integration }
\end{array}\right.
\end{aligned}
$$

There are several ways to implement an algorithm that computes the forward model for RF tomography. A fast yet optimized method is presented next. We shall point out that the formulas and procedures presented here are not suited for the computation of a single Green's function (the interested reader can consult $[7,10,28]$ ), but they are specifically designed for the matrix filling of $\mathbf{L}$ in (7) assuming $D$ is composed of a set of horizontal slices.

Due to the symmetry of the half-space geometry, one can show that

$$
\underline{\mathbf{S}}\left(\mathbf{r}, \mathbf{r}^{\prime}\right)=\underline{\mathbf{S}}\left(0,0, z, x^{\prime}-x, y^{\prime}-y, z^{\prime}\right)
$$

and similarly

$$
\underline{\mathbf{S}}\left(\mathbf{r}, \mathbf{r}^{\prime}\right)=\underline{\mathbf{S}}\left(x-x^{\prime}, y-y^{\prime}, z, 0,0, z^{\prime}\right) \text {. }
$$

With a proper manipulation, one can easily recast (40) as

$$
\begin{aligned}
& \underline{\mathbf{S}}_{R}^{a a, e e}\left(0,0, z, x^{\prime}-x, y^{\prime}-y, z^{\prime}\right) \\
& \cong \sum_{k_{x}=-K_{x}}^{K_{x}} \sum_{k_{y}=-K_{y}}^{K_{y}} A^{a a, e e} \underline{\mathbf{G}}^{a a, e e} e^{-i k_{x} x^{\prime}-i k_{y} y^{\prime}}
\end{aligned}
$$

and (52) as

$$
\begin{aligned}
& \underline{\mathbf{S}}^{a e, e a}\left(0,0, z, x^{\prime}-x, y^{\prime}-y, z^{\prime}\right) \\
& \cong \sum_{k_{x}=-K_{x}}^{+K_{x}} \sum_{k_{y}=-K_{y}}^{+K_{y}} A^{a e, e a} \tilde{\mathbf{G}}^{a e, e a} e^{-i k_{x} x^{\prime}-i k_{y} y^{\prime}}
\end{aligned}
$$

which can be solved using 2D-FFT routines. Similarly, the functions

$$
\begin{aligned}
& \underline{\mathbf{S}}_{R}^{a a, e e}\left(x-x^{\prime}, y-y^{\prime}, z, 0,0, z^{\prime}\right) \\
& =\sum_{k_{x}=-K_{x}}^{+K_{x}} \sum_{k_{y}=-K_{y}}^{+K_{y}} A^{a a, e e} \underline{\mathbf{G}}^{a a, e e} e^{+i k_{x} x+i k_{y} y}
\end{aligned}
$$

$$
\begin{aligned}
& \underline{\mathbf{S}}^{a e, e a}\left(x-x^{\prime}, y-y^{\prime}, z, 0,0, z^{\prime}\right) \\
& =\sum_{k_{x}=-K_{x}}^{+K_{x}} \sum_{k_{y}=-K_{y}}^{+K_{y}} A^{a e, e a} \underline{\mathbf{G}}^{a e, e a} e^{+i k_{x} x+i k_{y} y}
\end{aligned}
$$

can be effectively solved using 2D-IFFT routines.

Accordingly, the region $D$ is divided in a set of horizontal slices, each one having extension $D_{x}, D_{y}$, respectively (possibly squared), and step size $2 \delta$. For each slice, the corresponding discretized Fourier domain will have extension $K_{x, y}=\pi / \delta$.

Then, the dyadic functions are evaluated for each slice using (59) or (60) and the symmetric relations (55), (56). With proper care, these routines can be used to compute effectively, in parallel, and without appreciable redundancy, equations (18), (20) and (23).

\section{Computational Analysis}

A qualitative computational analysis of the forward model (filling of $\mathbf{L}$ ) is:

$$
\begin{aligned}
& O \propto 9 N_{f}\left[\left(N_{F F T}\right)^{2} \log \left(N_{F F T}\right)\right] \times \\
& {\left[N_{\tilde{z}}\left(N_{\tilde{z}}+N_{\bar{z}}+N_{z_{R x}}+N_{z_{T x}}\right)+\right.} \\
&\left.N_{\bar{z}}\left(N_{z_{R x}}+N_{z_{T x}}+N_{\tilde{z}}\right)+N_{z_{T x}} N_{z_{R x}}\right] \\
&+(9 P)^{3} N_{f}+(9 P)^{2} N_{f} \\
& \cong K_{1}\left[\left(N_{F F T}\right)^{2} \log \left(N_{F F T}\right)\right]+K_{2} P^{3}
\end{aligned}
$$

where $N_{f}$ is the number of frequencies, $N_{\tilde{z}}$ is the number of horizontal layers used to define the irregular terrain, $N_{\bar{z}}$ is the number of horizontal layers used to define the domain of investigation $D, N_{Z_{R x}}$ is the number of horizontal layers where receivers are located, $N_{Z_{T X}}$ is the number of horizontal layers where transmitters are located, $N_{F F T}$ is the number of bins used to define the Fourier space, and $K_{1}, K_{2}$ are two proportionality constants. Note that the algorithm utilizes $K_{1}$ independent 2D-FFT instances, and $K_{2}$ independent matrix inversions: with the use of parallel processing, the overall CPU time can be dramatically reduced.

The inversion of $\mathbf{L}$ depends upon the algorithm, but its computational complexity is generally much less than $\left(N_{E} N_{V}\right)^{3}$, particularly for sparse regularization algorithms such as FISTA. Note that the computational complexity can be maintained low, since in general $N_{z_{T x}}=N_{z_{R x}}=1$. In 
particular, from (61) it is clear the importance of keeping $N_{\bar{z}}$ low, i.e., horizontal layers are much faster to be computed rather than vertical cuts.

Using a standard PC, filling $\mathbf{L}$ required about 10 minutes using numerical Green's function, and about 1 minute using flat surface. The FISTA algorithm required less than 1 minute to provide the reconstructed images. However, neither GPU acceleration, nor fast matrix inversion was used.

\section{ACKNOWLEDGMENT}

The authors are thankful to Mr. Timothy Poth, Dr. Braham Himed, Air Force Research Laboratory, and to Dr. Jon A. Sjogren, Air Force Office of Scientific Research, for sponsoring and funding this research.

\section{REFERENCES}

[1] L. Lo Monte, D. Erricolo, F. Soldovieri, M.C. Wicks, "Radio Frequency Tomography for Tunnel Detection," IEEE Trans. Geosci. Remote Sens., Vol. GRS-48, No. 3, pp. 1128-1137, Mar. 2010.

[2] P. Meincke, "Linear GPR Inversion for Lossy Soil and a Planar Air-Soil Interface," IEEE Trans. Geosci. Remote Sens., Vol. GRS-39, No. 12, pp. 2713-2721, Dec. 2001.

[3] F. Soldovieri, J. Hugenschmidt, R. Persico and G. Leone, "A linear inverse scattering algorithm for realistic GPR applications," Near Surface Geophysics, vol. 5, no. 1, pp. 29-42, 2007.

[4] T. J. Cui, Y. Quin, Y. Ye, J. Wu, G. Wang, W. C. Chew, "Efficient Low-Frequency Inversion of 3-D Buried Objects With Large Constrasts," IEEE Trans. Geosci. Remote Sens., Vol. GRS-44, No. 1, pp.3-9, Jan. 2006.

[5] T. J. Cui, and W. C. Chew, "Diffraction Tomographic Algorithm for the Detection of Three-Dimensional Objects Buried in a Lossy Half-Space," IEEE Trans. Antennas Propag., Vol. AP-50, No. 1, pp. 42-49, Jan. 2002.

[6] W. C. Chew, J. M. Jin, E. Michielssen, J. Song, Fast and Efficient Algorithms in Computational Electromagnetics, Artech House, Boston, MA, 2001.

[7] W. C. Chew, Waves and Fields in Inhomogeneous Media, IEEE Press, New York, NY, 1995.

[8] T. B. Hansen, and P. M. Johansen, "Inversion Scheme for Ground Penetrating Radar that Takes Into Account the Planar Air-Soil Interface," IEEE Trans. Geosci. Remote Sens., Vol. GRS-38, No. 1, pp. 496-506, Jan. 2000.

[9] M. S. Zhdanov, Geophysical Electromagnetic Theory and Methods, Vol. 43, Elsevier Science, Amsterdam, the Netherland, 2009.

[10] W. C. Chew, M. S. Tong, B. Hu, Integral Equation Methods for Electromagnetic and Elastic Waves, Synthesis Lectures on Computational Electromagnetics, Morgan\&Claypool Publishers, 2009.

[11] M. S. Zhdanov, Geophysical Inverse Theory and Regularization Problems, Methods in Geochemistry and Geophysics, Vol. 36, Elsevier, Amsterdam, 2002.

[12] M. Bertero, and P. Boccacci, Introduction to Inverse Problems in Imaging, Institute of Physics Ltd, London, UK, 2002.

[13] R. Tibshirani, "Regression Shrinkage and Selection via the Lasso," $J$. Royal. Statist. Soc B., Vol. 58, No. 1, pp 267-288, 1996.

[14] A. Beck, M. Teboulle, "A Fast Iterative Shrinkage-Thresholding Algorithm for Linear Inverse Problems," SIAM J. Imaging Sci., Vol. 2, pp. 183-202, 2009.

[15] L. Lo Monte, J. T. Parker, "Sparse Reconstruction Methods in RF Tomography for Underground Imaging," IEEE Waveform Diversity and Design Conference 2010, Niagara Falls, ON, Aug. 2010.

[16] L. Lo Monte, D. Erricolo, F. Soldovieri, M. C. Wicks, "RF Tomography for Belowground Imaging of Extended Targets and Close-in Sensing" IEEE Geosci. Remote Sens. Lett.., Vol. GRSL-7, No. 3, pp. 496-500, Jul. 2010.
[17] J. De Zaeytijd, A. Franchois, C. Eyraud, J. Geffrin, "Full-Wave ThreeDimensional Microwave Imaging With a Regularized Gauss-Newton Method - Theory and Experiment," IEEE Trans. Antennas Propag., Vol. AP-55, No. 11, pp. 3279-3292, Nov. 2007.

[18] I. Catapano, L. Crocco, T. Isernia, "Improved Sampling Methods for Shape Reconstruction of 3-D Buried Targets," IEEE Trans. Gesoci. Remote Sens., Volt GRS-46, No. 10, pp. 3265-3273, Oct. 2008.

[19] G. A. Meles, J. Van der Krik, S. A. Greenhalgh, J. R. Ernst, H. Maurer, A. G. Green, "A New Vector Waveform Inversion Algorithm for Simultaneous Updating of Conductivity and Permittivity Parameters From Combination Crosshole/Borehole-to-Surface GPR Data," IEEE Trans. Geosci. Remote Sens., Vol. GRS-48, No. 9, pp. 3391-3407, Sep. 2010.

[20] L. Lo Monte, L. K. Patton, M. C. Wicks, "Mitigation of Coupling in RF Tomography with Applications to Belowground Imaging," IEEE International Radar Conference, Washington, DC, May 10-14 2010.

[21] B. Guan, J. F. Zhang, X. Y. Zhou, T. J. Cui, "Electromagnetic Scattering from Objects Above a Rough Surface Using the Method of Moments with Half-Space Green's Function," IEEE Trans. Geosci Remote Sens., Vol. GRS-47, No. 10, pp. 3399-3405, Oct. 2009.

[22] Y. Altuncu, I. Akduman, O. Ozdemir, A. Yapar, "Numerical Computation of the Green' Function of a Layered Media With Rough Interfaces," Microwave \& Optical Tech. Lett., Vol. 19, No. 5, pp. 12041209, Mar. 2007.

[23] Y. Altuncu, A. Yapar, I. Akduman, "On the Scattering of Electromagnetic Waves by Bodies Buried in a Half-space with Locally Rough Interface," IEEE Trans. Geosci. Remote Sens., Vol. GRS-44, No. 6, pp. 1435-1443, Jun. 2006.

[24] C. A. Balanis, Advanced Engineering Electromagnetics, John Wiley and Sons, Hoboken, NJ, 1989.

[25] P. C. Hansen, Rank Deficient and Discrete Ill-Posed Problems, SIAM, Philadelphia, PA, 1998.

[26] A. D. Yaghjian, "Electric Dyadic Green's Functions in the Source Region,” Proc. IEEE, Vol. 68, pp. 248-263, 1980.

[27] G. Gao, C. Torres-Verdin, T. M. Habashy, "Analytical Techniques to Evaluate the Integrals of 3D and 2D Spatial Dyadic Green's Functions," Progress in Electromagnetic Research, PIER 52, pp. 47-80, 2005.

[28] T. J. Cui, W. C. Chew, A. A. Aydiner, Y. H. Zhang, "Fast-Forward Solvers for the Low-Frequency Detection of Buried Dielectric Objects," IEEE Trans. Geosci. Remote Sens., Vol GRS-41. No. 9, pp. 2026-2036, Sept. 2003.

[29] T. J. Cui, W. C. Chew, Fast Evaluation of Sommerfeld Integrals for EM Scattering and Radiation by Three-Dimensional Buried Objects, Research Report No. CCEM-34-97, Center of Compuational Electromagnetics, Departmnet of Electrical and Computer Engineering, University of Illinois at Urbana-Champaign, Dec. 161997.

[30] T. J. Cui, W.C. Chew, Efficient Method for the Near-Field Detection of Buried Dieletric and Conducting Objects, Research Report No. CCEM3-98, Center of Computational Electromagnetics, Department of Electrical and Computer Engineering, University of Illinois at UrbanaChampaign, Jan. 281998.

[31] T. J. Cui, W. C. Chew, Fast Algorithm for Electromagnetic Scattering by Buried 3D Dielectric Objects of Large Size, Center of Computational Electromagnetics, Department of Electrical and Computer Engineering, University of Illinois at Urbana-Champaign, Aug. 31998.

[32] T. J. Cui, W. C. Chew, "Fast Evaluation of Sommerfeld Integrals for EM Scattering and Radiation by Three-Dimensional Buried Objects," IEEE Trans. Geosci. Remote Sens., Vol. GRS-37, No. 2, pp. 887-900, Mar. 1999.

[33] T. J. Cui, W. C. Chew, A. A. Aydiner, D. L. Wright, D. V. Smith, J. D. Abraham, "Numerical Modeling of an Enhanced Very Early Time Electromagnetic (VETEM) Prototype System," IEEE Antennas Propag. Mag., Vol. 42, No. 2, pp. 17-56, Apr. 2000.

[34] X. F. Liu, B. Z. Wang, S. Q. Xiao, "Electromagnetic Subsurface Detection Using Subspace Signal Processing and Half-Space Green's Function," Progress in Electromagnetics Research, PIER 98, pp. 315331, 2009.

[35] G. R. Olhoeft, "Electrical Properties of Rocks" [book auth.] Y. S. Touloukian, W. R. Judd and R. F. Roy, Physical Properties of Rocks and Minerals. Hemisphere Publishing Corporation, pp. 257-329, New York, NY 1989. 
[36] E. I. Parkhomenko, Electrical Properties of Rocks, Plenum Press, New York, NY, 1967.

[37] A. Giannopoulos, GPRMAX Simulator, www.gprmax.org

[38] D. W. Winters, B. D. Van Veen, S. C. Hagness, "A Sparsity Regularization Approach to the Electromagnetic Inverse Scattering Problem," IEEE Trans. Antennas Propag., Vol. AP-58, No.1, pp. 145154, Jan. 2010.

[39] M. A. Ali, M. Moghaddam, "3D Nonlinear Super-Resolution Mirowave Inversion Technique using Time-Domain Data," IEEE Trans. Antennas Propag., Vol. AP-58, No. 7, pp. 2327-2336, Jul. 2010.

[40] A. Tabatabaeenejad, M. Moghaddam, "Inversion of Subsurface Properties of Layered Dielectric Structures With Random Slightly Rough Interfaces Using the Method of Simulated Annealing," IEEE Trans. Geosci. Remote Sens., Vol. GRS-47, No. 7, pp. 2035-2046, Ju. 2009.

[41] P. Imperatore, A. Iodice, D. Riccio, "Electromagnetic Wave Scattering From Layered Structures With an Arbitrary Number of Rough Interfaces," IEEE Trans. Geosci. Remote Sens., Vol. GRS-47, No. 4, pp. 1056-1072, Apr. 2009

Lorenzo Lo Monte (S'05-M'10) received his B.Sc. and M.Sc. (both summa cum laude) in Telecommunications engineering from University of Rome "Tor Vergata", Rome, Italy in 2003 and 2005, respectively, and his Ph.D. in Electrical Engineering at the University of Illinois at Chicago (UIC), Chicago, IL, USA, in 2009.

In 2007 he worked as a contractor for the Air Force Research Laboratory / Sensor Directorate on RF sensing and electromagnetic imaging. From 2007 to 2008, he worked at PCTEL Inc. as antenna engineer, developing a new family

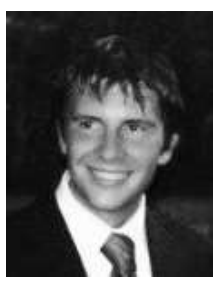
of WiMax BTS sector panels. Since March 2008 he is a radar / RF systems engineer at General Dynamics Information Technology in Dayton, $\mathrm{OH}$, working as a contractor for Air Force Research Laboratory / RYMD branch at Wright-Patterson Air Force Base, Dayton, OH. $\mathrm{He}$ was visiting scholar at Rensselaer Polytechnic Institute, Troy, NY, and Wright State University, Dayton $\mathrm{OH}$, performing research in inverse problems for radar imaging. His main research interests include radar imaging, tomography, subsurface sensing and inverse scattering.

Francesco Soldovieri (M'10) received the Laurea degree in electronic engineering from University of Salerno in 1992, and the Ph.D. degree in electronic engineering in 1996 from University of Naples "Federico II".

In 1993, he joined the Electromagnetic Research Group of University of Naples and in 1998/1999 he held a Postdoctoral Fellowship at the same University. Since 2001 he is Researcher at the Institute for the Electromagnetic Sensing of the Environment of the Italian National Research

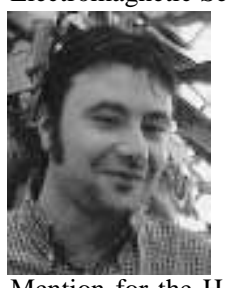
Council (IREA-CNR). Since 2006 he is Senior Researcher at IREA. He was General Chair of the International Workshop on Advanced Ground Penetrating Radar 2007. Since 2002, he is involved in the Technical Committees of the GPR Conference and IWAGPR Workshop. He has been Special Guest Editor for Issues on Journal of Applied Geophysics, Near Surface Geophysics, Advances in Geosciences. He was awarded the 1999 Honorable
A. Wheeler Applications Prize Paper Award of the IEEE Mention for the $\mathrm{H}$

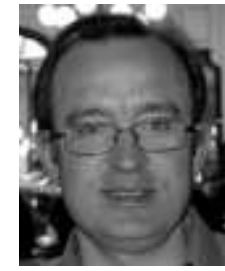
Antennas and Propagation Society. $\mathrm{He}$ is listed in Who's Who in Science and Engineering, 2006-2007 and 2007-2008. His main scientific interests include electromagnetic diagnostics, inverse scattering, GPR applications, antenna diagnostics and characterization, security applications.

Danilo Erricolo (S'97-M'99-SM'03) received the
Laurea degree of Doctor (summa cum laude) in electronics engineering from the Politecnico di Milano, Milano, Italy, in 1993 and the Ph.D. degree in electrical engineering and computer science from the University of Illinois at Chicago (UIC) in 1998. He is currently an Associate Professor in the Department of Electrical and Computer Engineering, UIC, where he is also the Associate Director of the Andrew Electromagnetics Laboratory. His research interests are primarily in the areas of wireless communications, electromagnetic scattering, and electromagnetic compatibility. His research activity has been supported by the Department of Defense and the National Science Foundation. He has authored or co-authored more than 150 publications in refereed journals and international conferences. He was twice the recipient of both the Andrew Foundation Fellowship and the Beltrami Foundation Fellowship, and a U.S. Air Force Summer Faculty Fellow in 2009. Dr. Erricolo is a member of Eta Kappa $\mathrm{Nu}$ and was elected a Full Member of the U.S. National Committee of the International Union of Radio Science (USNC-URSI) Commissions B and E. He served for USNC-URSI as Secretary (2004-2005), Vice-Chair (2006-2008) and Chair (2009-2011) of Commission E and Chair of the Ernest K. Smith Student Paper Competition (2009-present). He is an Associate Editor for the IEEE Antennas and Wireless Propagation Letters. He also has served as Vice-Chair of the Local Organizing Committee of the XXIX URSI General Assembly (Chicago, IL USA, Aug. 7-

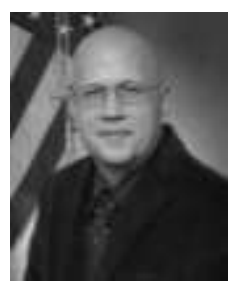
16, 2008) and as General Chair of the 2012 IEEE Antennas and Propagation International Symposium/USNC National Radio Science Meeting (Chicago, IL USA, July 8-14, 2012).

Michael C. Wicks (S'81-M'89-SM'90-F'98) received the B.Sc. degree (1981) from Rensselaer Polytechnic Institute, Troy, NY, and the M.Sc. (1985) and Ph.D. (1995) degrees from Syracuse University, Syracuse, NY in electrical engineering. He earned a Master of Arts in Public Administration in 2000 from the Maxwell School of Syracuse University.

Dr. Wicks retired from the U.S. Air Force Research Laboratory Sensors Directorate after a 30 year career, and joined the Department of Electrical and Computer Engineering at the University of Dayton. He is also a Distinguished Research Scientist at the University of Dayton Research Institute Sensor Systems Division. He has authored over 300 papers, reports, and patents. His technical expertise encompasses space-time adaptive processing, advanced algorithm development, ultra-wideband radar, polarimetric sensor signal processing, inverse synthetic aperture radar imaging, knowledge-base applications to radar signal processing, concealed weapons detection, groundpenetrating radar, bistatic radar, and radar systems engineering. Dr. Wicks holds 15 U.S. patents, and has authored or co-authored three books, many book chapters, and over 300 journal, conference, and technical papers. Additionally, Dr. Wicks participates in numerous national and international panels, committees, and working groups, including NATO and other multinational organizations.

Dr. Wicks serves on the Board of the Central Association for the Blind and Visually Impaired, the New York State Mohawk Valley Water Authority, the Advisory Board to the Department of Engineering, Computer and Physical Sciences at Mohawk Valley Community College, the Advisory Board at the Rochester Institute of Technology Electrical Engineering Department. He is an AFRL and an IEEE Fellow, 2009 winner of the IEEE Warren D. White Award for Excellence in Radar Engineering and 1998 recipient of the IEEE Fred Nathanson Award to the Young Engineer of the Year. Dr. Wicks also serves as an adjunct faculty member at the University College London, United Kingdom. 